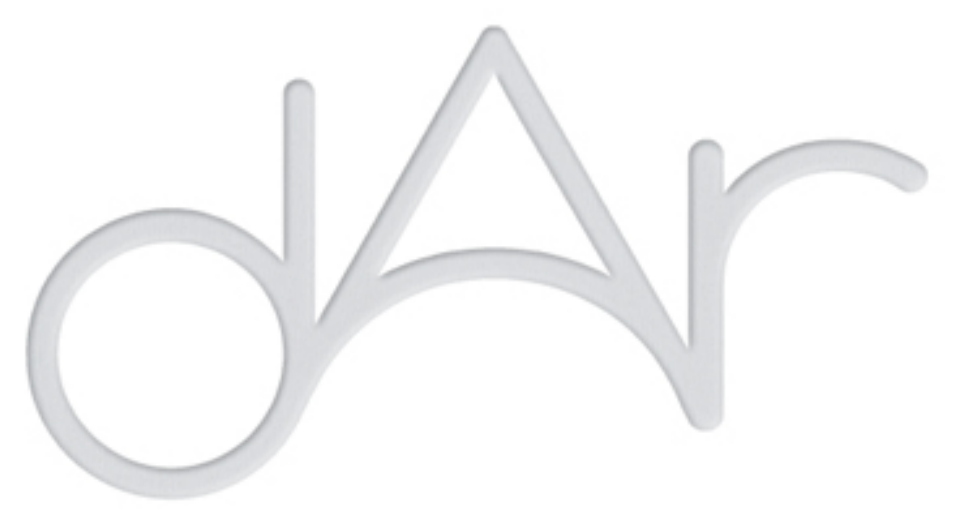

\title{
Consommation du patrimoine hydraulique par le activités touristiques: cas de la haute vallee du Draa
}

\author{
Autor(es): $\quad$ Abderrahmane, Oujamaa \\ Publicado por: Imprensa da Universidade de Coimbra \\ URL \\ persistente: URI:http://hdl.handle.net/10316.2/44428 \\ DOI: $\quad$ DOI:https://doi.org/10.14195/2182-844X_5_7 \\ Accessed : $\quad$ 26-Apr-2023 13:51:55
}

A navegação consulta e descarregamento dos títulos inseridos nas Bibliotecas Digitais UC Digitalis, UC Pombalina e UC Impactum, pressupõem a aceitação plena e sem reservas dos Termos e Condições de Uso destas Bibliotecas Digitais, disponíveis em https://digitalis.uc.pt/pt-pt/termos.

Conforme exposto nos referidos Termos e Condições de Uso, o descarregamento de títulos de acesso restrito requer uma licença válida de autorização devendo o utilizador aceder ao(s) documento(s) a partir de um endereço de IP da instituição detentora da supramencionada licença.

Ao utilizador é apenas permitido o descarregamento para uso pessoal, pelo que o emprego do(s) título(s) descarregado(s) para outro fim, designadamente comercial, carece de autorização do respetivo autor ou editor da obra.

Na medida em que todas as obras da UC Digitalis se encontram protegidas pelo Código do Direito de Autor e Direitos Conexos e demais legislação aplicável, toda a cópia, parcial ou total, deste documento, nos casos em que é legalmente admitida, deverá conter ou fazer-se acompanhar por este aviso.

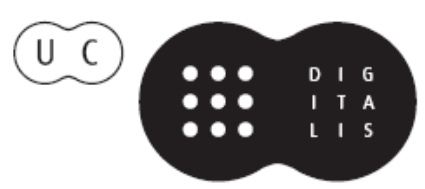



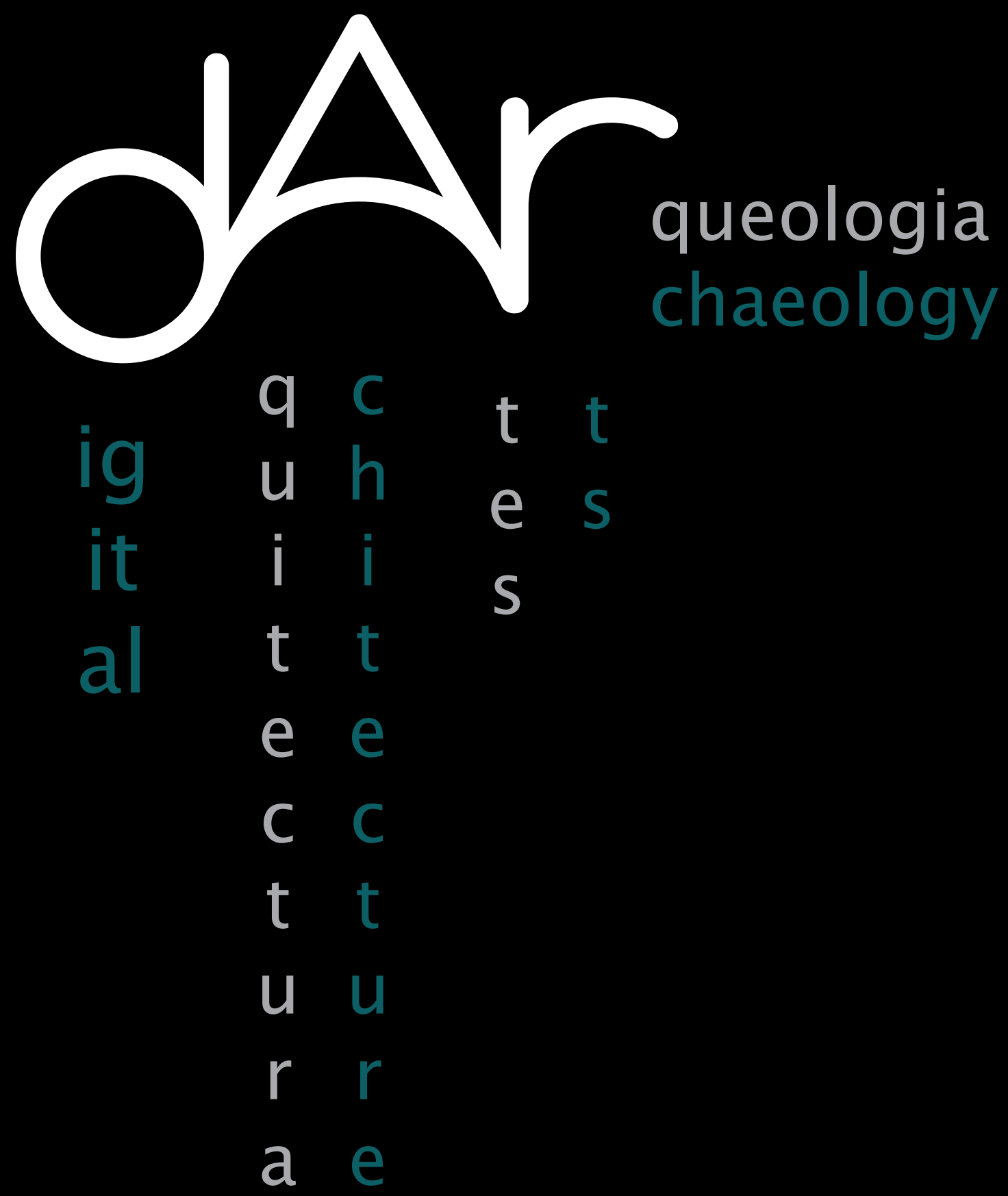

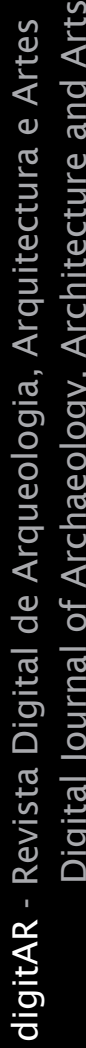




\title{
CONSOMMATION DU PATRIMOINE HYDRAULIQUE PAR LE ACTIVITÉS TOURISTIQUES:
}

Cas de la Haute Vallee du DRAa

\author{
Dr. Oujamaa Abderrahmane \\ Cadre supérieur (ONE, Casablanca)/CIERSGSDDOM
}

\section{Résumé}

D'une capacité d'hébergement touristique faible au niveau national durant les années soixante et parmi les cinq premières zones d'aménagement touristique choisis par l'Etat (Plan 1965-1967), la Province d'Ouarzazate est devenue parmi les destinations touristiques du Maroc plus programmées dans les flux touristiques internationales. En effet, dans le cadre d'un développement socio-économique harmonieux, l'Etat a accordé une grande importance aux régions des zones sud-atlasiques. Le Grand-Sud, englobant les deux Provinces Ouarzazate et Errachidia, a bénéficié d'un schéma d'aménagement touristique depuis 1978 qui a permis de tracer les grandes lignes du développement de cette zone.

Nous essayons à travers cet article de montrer l'importance de la consommation du patrimoine hydraulique par le secteur touristique dans la zone aride, et comment peut-on encourager un secteur consommable de l'eau avec le développement et le bien-être de la population sans pour autant impacter négativement le milieu naturel et le patrimoine culturel?

Mots-clé: Tourisme, Espace, Développement, Ouarzazate, Oasis, Consommation de l'eau, Environnement.

\section{Introduction}

Le tourisme a été choisi en tant que locomotive de développement grâce aux opportunités d'emploi qu'il génère et les devises qu'il procure, ainsi que l'ouverture qu'il favorise en tant que vecteur de la modernité et de la valorisation du patrimoine culturel de la zone.

Le développement économique de la région d'une part et sa richesse patrimoniale en termes de monuments, d'ouvrages d'art ou de paysages 
d'autre part, restent deux éléments inséparables. Cette situation apparaît plutôt comme une opportunité en vue d'une meilleure répartition du développement économique à travers la région. En effet, bien qu'il soit possible de concentrer dans un même point richesse architecturale, beauté des sites naturels et densité du tissu économique, rares sont les régions qui peuvent rassembler simultanément tous ces atouts.

Et malgré l'importance du secteur touristique dans l'aménagement du territoire en général et l'organisation des espaces régionaux, son évolution dans des régions présente des déséquilibres même au sein des milieux présentant des caractéristiques plus au moins identiques. L'analyse des déséquilibres régionaux, la valorisation du patrimoine à des fins touristiques et l'enjeu environnemental relatif à la demande touristique en eau potable constituent les axes de réflexion de cette intervention à travers le cas de la haute vallée du Drâa (Province d'Ouarzazate) qui a constitué un espace préféré de nos recherches ${ }^{1}$.

En effet, la région de Drâa-Tafilelt, a fait l'objet d'une valorisation touristique depuis les années $80^{2}$. Cette région, regroupant les deux provinces Ouarzazate et Errachidia, constitue l'une des destinations touristiques marocaines à caractère culturel et espace rural recherché.

En effet, le développement touristique dans cette région présaharienne ne signifie pas uniquement le renforcement du tourisme culturel et de désert, mais pouvait contribuer à :

$\checkmark$ l'atténuation des disparités régionales;

$\checkmark$ diversification et complémentarité du produit touristique ;

$\checkmark$ développement économique de cette zone.

Ace stade, il s'agit d'identifier les atouts de la région, en termes de moyens de production de valeur économique en se basant sur un diagnostic du produit touristique, mode de vie, infrastructures et commercialisation etc.

\footnotetext{
1 A) Oujamaa Abderrahmane, 1999, Tourisme et l'espace - cas de Ouarzazate et Errachidia entre le choix des décideurs et la réalité actuelle, Thèse de Troisième Cycle (DES), Université Mohamed V - Faculté des Lettres et des Sciences Humaines - Rabat, Unité de formation et de recherche «Aménagement de l'espace régional», 410p.

B) Oujamaa Abderrahmane, 2007, Activités touristiques et consommation de l'eau l'eau dans les oasis du sud: cas Haut vallée du Draa, Doctorat National, Université Hassan II - Faculté des Lettres et des Sciences Humaines - Mohammadia, Unité de formation et de recherche «Eau et Civilisation», pp 310.

2 L`aménagement touristique, 1978; Ouarzazate - Errachidia.
} 
Le potentiel touristique et culturel en termes de rayonnement national, régional et international est souvent sous-estimé ou ne fait éventuellement l'objet d'aucune étude particulière. Il apparaît aujourd'hui qu'un intérêt croissant pour le patrimoine de cette région se fait à jour de la part des touristes étrangers comme des habitants de la région et les acteurs concernés. $\mathrm{Au}$ vu du poids économique croissant que représente le secteur touristique au Maroc et particulièrement pour la région de Drâa et Tafilalet, ce patrimoine doit aujourd'hui être considéré comme un facteur de développement de premier plan, mais aussi comme un moyen de redéploiement économique de la région.

A travers cette intervention, l'approche vise essentiellement à développer ces axes suivants:

1. Naissance et évolution du secteur touristique du Grand-Sud ;

2. Les atouts de la région et aménagement de l'espace touristique;

3. Les impacts du secteur touristique ;

4. Vers une valorisation du patrimoine culturel;

\section{D'un tourisme spontané à un tourisme permanent}

Pour synthétiser la naissance et l'évolution de cet espace touristique, les étapes méritent d'être soulignées :

- Etape 1, le Drâa et le Tafilelt recevaient un tourisme spontané. Cette période a commencé avec l'époque coloniale et va jusqu'à la moitié des années soixante. L'infrastructure touristique embryonnaire telle que les gîtes d'étapes le long des vallées du Drâa et du Ziz a fait son apparition. A la fin de 1964, cette région ne représentait que 1,65\% de la capacité hôtelière totale.

- Etape 2 l'Etat a accordé une grande importance au secteur touristique (plan triennal 1965-1967) considéré comme un moyen de développement local. Cinq Zones d'Aménagement Touristiques Prioritaire (Z.A.P) ont vu le jour dont fait partie la région du Drâa-Tafilelt. L'Etat a mis en place une infrastructure hôtelière et routière (rocade-sud), en plus de la création des aéroports d'Ouarzazate et d'Errachidia. Et malgré les encouragements et les facilités accordées aux investisseurs privés (code des investissements 19651973) on a constaté un engagement faible et timide de ces derniers dans cette zone.

- Etape 3, se caractérise par le désengagement de l'Etat (le plan 1978-1980). 
Ceci a causé une stagnation de la croissance touristique dans la région. Ce n'est qu'au milieu des années quatre-vingt que le secteur touristique va connaître un réel démarrage grâce aux interventions du secteur privé.

Le lancement par l'Etat du tourisme dans la région s'est appuyé sur une étude d'aménagement touristique représentant la base de programmation et de planification de l'infrastructure touristique dans la région (fig $\mathrm{n}^{\circ} 1$ ). Ce programme, basé essentiellement sur les richesses patrimoniales des localités, a accordé depuis le début une grande importance à la distinction entre les pôles principaux, les pôles secondaires, les relais et enfin les centres d'accueil.

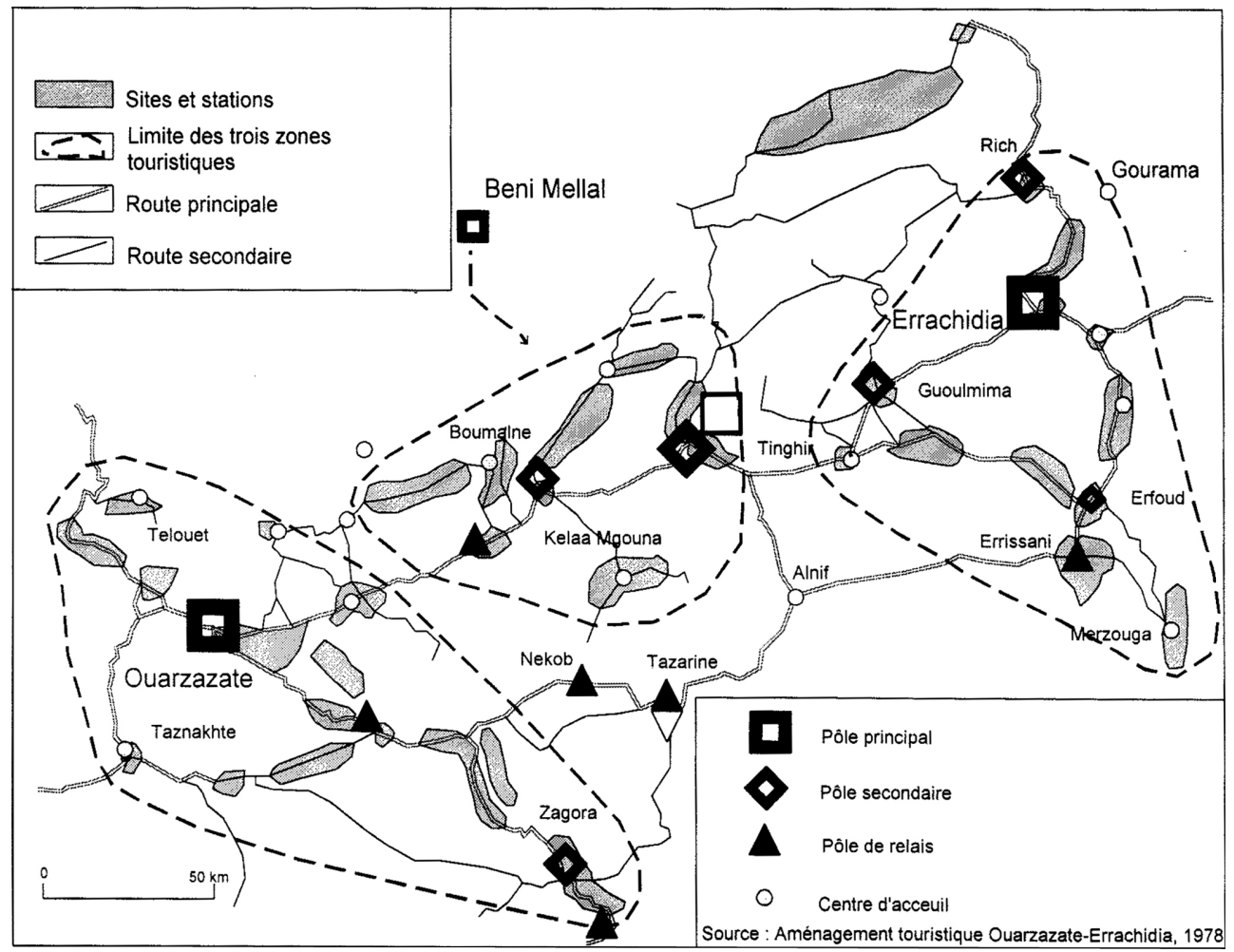

Fig. 1 - Armature touristique du Sud-intérieur - 1978

Chefs-lieux des deux provinces, les villes d'Ouarzazate et d'Errachidia constituent des pôles principaux. Ceci leur permet de jouer le rôle d'accueil 
et de distribution des flux touristiques vers les différents points de la région. En même temps, le projet a prévu la mise en place d'une infrastructure de base au niveau des autres pôles dans le but d'atteindre une harmonie de l'unité que constitue la région.

A travers cette classification, l'unité touristique du Sud intérieur s'articule autour de trois axes principaux :

$\checkmark$ A l'Est la zone d'Errachidia

$\checkmark$ Au centre la vallée du Dadès-Todra et le massif du Saghro.

$\checkmark$ A l'ouest la zone d'Ouarzazate et du Drâa.

Cette organisation devait permettre une bonne répartition de l'infrastructure d'hébergement et une utilisation rationnelle de l'espace et de ces unités.

La constante principale du projet d'aménagement touristique du Sud intérieur était d'assurer un équilibre en matière d'infrastructure au sein de la région. Mais l'analyse de la répartition des infrastructures et la prise en compte de la dynamique de développement du tourisme dans cette région montre un déséquilibre entre le pôle d'Ouarzazate qui s'affirme comme un futur pôle touristique et la région d'Errachidia où l'activité touristique se développe timidement. D'où l'intérêt de s'interroger sur le pourquoi de ce déséquilibre alors que les programmations visaient un développement équilibré entre les deux pôles. Réfléchir sur les raisons du développement déséquilibré du tourisme dans cette région, c'est aborder le problème des facteurs de localisation des activités touristiques à partir de cas concrets, ainsi que l'enjeu environnemental de ce secteur.

\section{Aspects du déséquilibre des infrastructures touristiques}

Le Sud intérieur détient une place non négligeable dans la capacité hôtelière nationale depuis la deuxième moitié des années quatre-vingt (fig. $n^{\circ} 2$ ). En effet, deux étapes dans l'évolution de la capacité hôtelière se distinguent :

- Une phase avant 1984, la capacité hôtelière se caractérise par une croissance lente : omission de l'intérieur.

- Après 1984, on enregistre une forte croissance qui s'explique par l'arrivée, pour la première fois, des grandes chaînes hôtelières dans la région.

Cette évolution de la capacité hôtelière dans la région est liée au processus 
que connaît l'espace touristique marocain qui a enregistré des changements considérables tels que le déplacement du pôle d'attraction du Nord vers le sud et le recul sensible mais encore faible de la part de la côte au cours des années 1990.

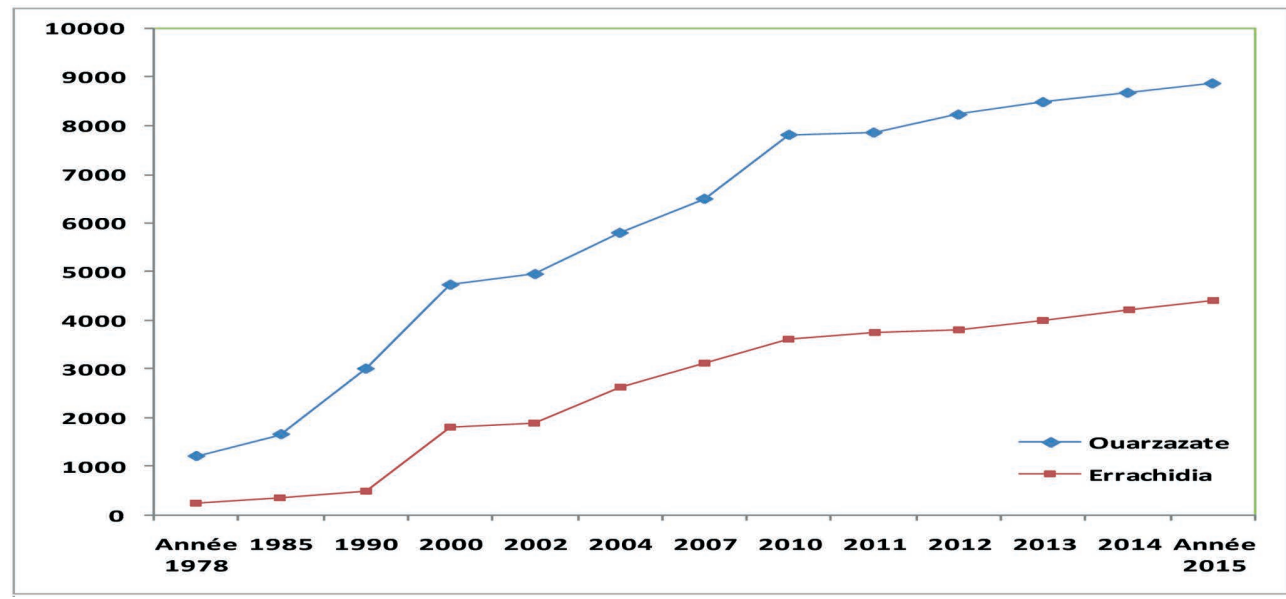

Fig. 2 - Evolution de la capacité hôtelière au Sud-intérieur

La structure hôtelière se caractérise par une dominance des hôtels de haut standing et l'absence de certaines catégories (RT, VVT) surtout à Errachidia. La part des catégories 4 - 5 étoiles dépasse largement la moitié de l'ensemble de la capacité hôtelière, soit $65,76 \%$.

Mais, depuis la fin de la décennie 80 , on assiste à une diversification de la structure d'hébergement. Il s'agit, de la création des résidences touristiques, de nombreux campings et d'auberges dans le but de répondre aux besoins du tourisme individuel et celui du désert (tourisme rural). C'est le cas également des auberges qui ont fait leur apparition au niveau de la zone dans le cadre des efforts visant le développement d'un tourisme intégrant la montagne et le désert. La quasi-totalité de ces unités se concentrent dans le massif du M'Goun et l'axe de Merzouga et Drâa.

Par ailleurs, l'augmentation de l'infrastructure touristique a suivi celle de la demande. Si on tient compte de la part des arrivées et des nuitées enregistrées dans les hôtels classés, on constate que la part du sud intérieur au niveau national est passée de 6,5\% en 1982 à $11 \%$ en 2000. Mais la durée de séjour ne dépasse guère 1,6 jour (tourisme de passage). Il ressort que l'évolution de la demande touristique est très irrégulière; mais l'année 1985 constitue 
un tournant dans l'évolution de la demande dans le sud intérieur. Cette dynamique du tourisme dans le sud intérieur est liée à des flux touristiques étrangers et instables. De plus le sud constitue un arrière-pays des grands pôles touristiques au niveau national comme Agadir et Marrakech.

Le tourisme international représente 95,6\% des flux. Quatre nationalités d'origine européenne (France, Allemagne, Espagne, Italie) représentent $81,7 \%$ de cette demande Ouarzazate détient les $3 / 4$ de cette demande, soit un taux de $78,3 \%$.

Parallèlement à l'infrastructure hôtelière, les activités de services liées au tourisme se diffusent sur de nombreux sites comme c'est le cas des unités localisées à Todra-Dadès, Aït Ben Haddou, Merzouga...

Mais la province d'Ouarzazate détient $77,7 \%$ de l'ensemble des unités de services enquêtées. Les 3/4 de ces unités sont apparues pendant les années quatre-vingt. Par contre Errachidia ne fixe que 22,3\% et le tiers a été créé pendant les années soixante et soixante-dix.

Les différenciations entre le Drâa et le Tafilelt apparaissent également au niveau des agences de voyage et de transport touristique. Les unités de vente de produits artisanaux accompagnent à leur tour le développement que connaît le secteur touristique dans la région.

Dans la province d'Errachidia, on assiste à une augmentation du nombre des unités de services. Mais celles-ci n'ont pas suivi l'évolution touristique : 60\% de ces unités sont apparues avant les années quatre-vingt. Seul le transport touristique a enregistré une évolution significative (77,8\%).

\section{Répartition spatiale de l'offre et de la demande touristique: Pôle d'Ouarzazate vers la concentration}

La ville d'Ouarzazate concentre à elle seule $54,3 \%$ des arrivées dans la région en 2004. Dans la province d'Errachidia deux centres apparaissent : Erfoud et le chef lieu. Ils concentrent $90 \%$ des arrivées enregistrées dans les hôtels classés. Nous signalons qu'Erfoud a dépassé Errachidia et concentre plus de $50 \%$ de cette demande $(62,5 \%$ des arrivées en 2004$)$.

La répartition géographique des structures d'accueil hôtelières représente un important indicateur pour mesurer les inégalités spatiales en matière d'industrie touristique. Elle constitue également un facteur révélant les impacts de cette industrie (Pearce, 1987). La province d'Ouarzazate concentrait $75 \%$ des lits en 1972 . Mais, cette situation va s'aggraver par la suite pour distinguer trois zones (1977): 
- L'axe Ouarzazate-Drâa avec 49\% des lits;

- L'axe Dades-Todghra avec 29\%;

- La province d'Errachidia avec 22\%.

L'arrivée des investissements privés à partir des années quatre-vingt va accentuer ce déséquilibre. La province d'Ouarzazate détient désormais $81,9 \%$ de la capacité commercialisée. Par contre la ville d'Errachidia a enregistré un recul au profit d'Erfoud. De ce fait, les grandes lignes directrices du développement touristique programmé dans la région et qui visaient le développement de deux pôles principaux sont encore loin d'être réalisées.

La répartition de la capacité d'hébergement au niveau régional et provincial montre qu'une série de centres commencent à renforcer l'armature touristique, mais n'arrivent pas à atteindre actuellement le seuil défini dans le projet touristique. Ces centres ne comportent que quelques unités telles que Tazarine, Agdz, Alnif... Ces centres ont permis de découvrir le massif du Saghro avec une ouverture vers l'Est passant par la route reliant Agdz à Errissani. Ce rôle peut être joué par d'autres centres comme Rich et Goulmima pour découvrir le Haut Atlas à l'Est. Les centres qui se développent au sud (M'hamid, Tamegroute, Merzouga) contribuent au développement d'un tourisme du désert.

En ce qui concerne la répartition des auberges rurales on assiste à une fixation de ces unités dans certains points délimités, soit le long des gorges de Dadès-Todra qui permettent de pénétrer le massif du M'Goun ou dans l'espace saharien à côté des dunes de sable de Merzouga et M'hamid.

$\mathrm{Au}$ terme de l'analyse de la répartition spatiale des infrastructures d'hébergement et des activités de services liées au secteur touristique, on peut distinguer trois axes :

- L'axe de Drâa-Ouarzazate qui détient 66,2\% de la capacité d'hébergement et $59,6 \%$ des activités de services et de commerces (bazars);

- L'axe Dades-Todra avec $12,8 \%$ et $18,1 \%$;

- L'axe du Tafilelt avec seulement $21,0 \%$ et $22,3 \%$.

Une situation qui diffère de la répartition visée par le projet d'aménagement touristique qui prévoyait la répartition de la capacité d'hébergement suivante : 40,58\% à l'Ouest, $29,16 \%$ au centre et $30,26 \%$ à l'Est. Ce qui montre que l'axe Ouarzazate se développe au détriment des autres axes. 


\section{Les projets touristiques futurs renforcent ces inégalités}

La tendance à la concentration des infrastructures touristiques reste la caractéristique principale. Trois indicateurs nous permettent de confirmer cette constatation : les projets touristiques, surtout hôteliers, le projet d'aménagement du site du lac Ahmed El Mansour Ed-Dahbi ( Fig n4) et le tourisme de montagne qui a pris son essor dans la région d'Ouarzazate.

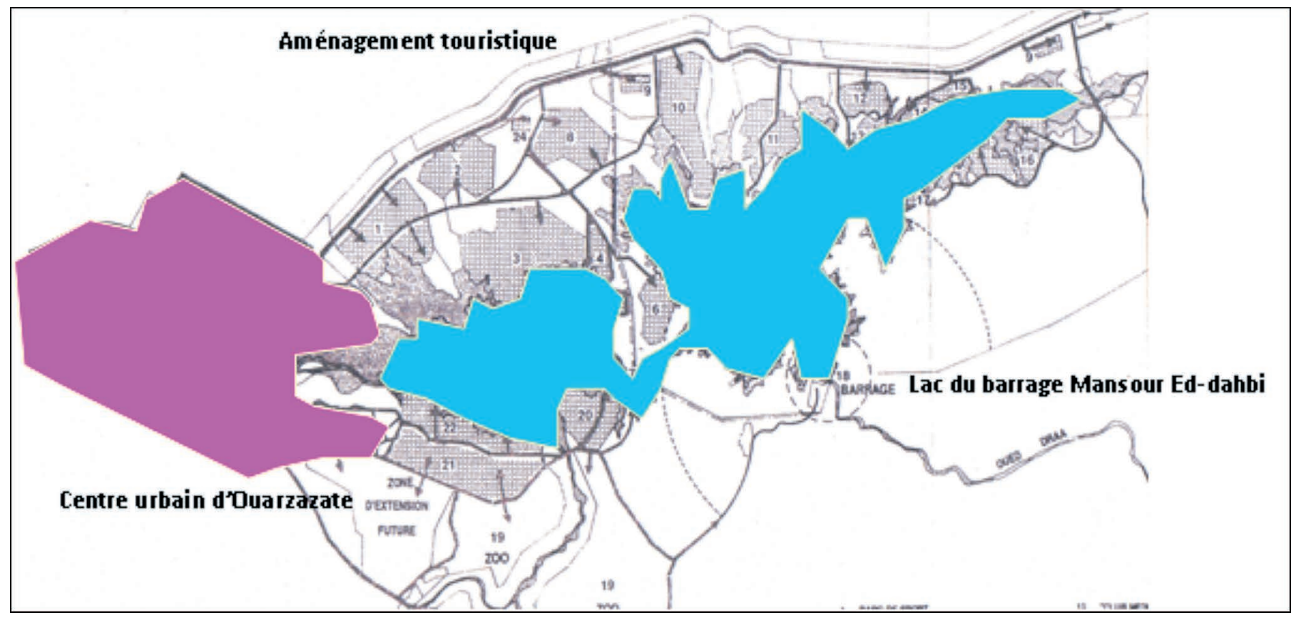

Fig. 3 - Projet d'aménagement du site du lac Ahmed El Mansour Ed-Dahbi

- Les hôtels en chantier et qui se concentrent à Ouarzazate ainsi que les projets touristiques en cours d'étude;

- Le projet d'aménagement du site du lac de barrage Ahmed El Mansour Ed-Dahbi couvre une superficie de 21.000 ha, dont celle réservée au projet touristique qui est de 1932 ha, pour une capacité d'hébergement de 30.000 lits à l'horizon 2010 (Royaume du Maroc, 1993). La réalisation de ce projet suit son chemin depuis l'inauguration du golf royal et du lotissement El Ahabab, projets qui ont attiré des entrepreneurs privés.

- Les projets visés dans le cadre de la vision 2020 surtout le label «Atlas et vallées»:

- Errachidia : selon les nouveaux territoires touristiques, elle fait partie de la région touristique «Atlas et vallées» pour laquelle on affiche de grandes ambitions à l'horizon 2020. Ce programme régional «Qariati» de développement intégré du tourisme rural et de nature, selon la 
vision 2020, au niveau de la province d'Errachidia, comporte 41 projets touristiques avec un investissement public de $81 \mathrm{MDH}$.

- Ouarzazate : Le Plan Solaire Maroc ambitionne de produire 2000 mégawatts d'énergie à l'horizon 2020 grâce aux énergies vertes comme le solaire, l'hydraulique ou l'éolien.

- La stratégie de développement des zones oasiennes et de l'arganier dont la région de Draa Tafilalet fait partie. La mise en œuvre de ce programme couvre différents secteurs, à savoir: les infrastructures de base, la santé, l'éducation et la formation, le tourisme et l'artisanat, l'agriculture et la gestion rationnelle des ressources hydriques...

V. Facteurs de localisation des infrastructures touristiques dans le Sud intérieur.

La localisation des implantations touristiques peut être définie comme l'ensemble des facteurs naturels, humains et économiques qui permet d'exploiter dans des conditions rentables des ressources originaires qui contribuent à une attraction de la fréquentation touristique. Dans le cas du Sud intérieur il n'est pas aisé de comprendre le pourquoi de ce déséquilibre tant les facteurs qui l'expliquent sont nombreux.

\section{1 - Produit touristique offert par le Drâa et le Tafilelt}

Le produit touristique du Drâa et du Tafilelt se caractérise par la diversification et la richesse de ses éléments.

- Climat, ressource touristique, mais élément d'explication non décisif

- L'espace formant les régions de Drâa et du Tafilelt s'étend des versants sud du haut Atlas au nord jusqu'aux confins algéro-marocains. Mais le climat intervient dans le choix des destinations touristiques, les formes d'accueil et les types de tourisme pratiqués. L'intervention de l'élément climatique se voit au niveau des étages bioclimatiques du nord au sud.

\section{- Patrimoine culturel}

Le patrimoine culturel du Drâa et du Tafilalt constitue l'un des aspects justifiant la demande touristique. Ce patrimoine contribue au développement 
des espaces touristiques mais détermine aussi la force d'attraction des régions. Le Drâa et le Tafilelt ont joué un rôle très important tout au long de l'histoire du Maroc, d'où la richesse culturelle: architecture, tradition ...,. Lorsqu'on parle de l'architecture traditionnelle du Sud intérieur, le Ksar et la Kasbah restent les formes d'habitat les plus présentes et le produit touristique le plus recherché. Ce symbole socio-économique se localise tout au long du Ziz, Rhris, Dades-Todra et Drâa. Mais il apparaît que la province d'Ouarzazate présente un grand avantage au niveau quantitatif et qualitatif. Selon l'inventaire du Ministère de la Culture, sur un ensemble de 670 éléments recensés, la province d'Ouarzazate englobe 569 contre 91 à Errachidia dont 293 kasbahs à Ouarazazate contre une vingtaine à Errachidia.

De plus, ce qui confirme cette richesse architecturale surtout à Ouarzazate c'est la création d'un Centre de Conservation du Patrimoine Culturel SubAtlasique au cœur de la ville même. Sans doute cette création montre de nouveau l'importance et la situation stratégique dont profite Ouarzazate et la richesse de ses environs. Cette création a réellement renforcé l'image touristique de la province d'Ouarzazate surtout au début de la restauration du patrimoine culturel dans certains villages (Aït Ben Haddou). A Errachidia, les interventions du centre restent encore limitées.

Sans pour autant oublier un nouveau produit touristique qui vient d'apparaître et qui méritent d'être exploité surtout dans une zone de la région d'Ouarzazate qui reste vierge : Circuit du dinosaure ${ }^{3}$. Le Conseil Provincial d'Ouarzazate avait signé une convention avec la commune rurale d'Iminoulaouen concernée. Pas moins de 1 million de dirhams seront débloqués pour la concrétisation de ce projet.

- Ouarzazate se prépare à devenir le leader cinématographique de l'Afrique Ouarzazate pourrait devenir le leader de l'industrie cinématographique en Afrique. C'est l'objectif d'une stratégie élaborée par le Conseil de la région de Souss-Massa-Drâa et le Centre cinématographique marocain (CCM). Présenté à SM le Roi Mohammed VI, lors de sa visite à Ouarzazate le 28 décembre 2007. Pour établir une vision stratégique pour le secteur, le conseil avait lancé une étude dont l'objectif est de garantir des retombées économiques et sociales pour la région et ses habitants.

3 Il est important de signaler qu'un musée aux standards internationaux sera mis en place d'ici deux ans pour abriter le Tazoudasaurus, du nom de Tazouda où il a été découvert (Commune rurale Imi n'Oulaoune) 
Cette stratégie adoptée a permis de dénombrer six chantiers à entreprendre pour une enveloppe globale estimée à 43 millions de dirhams. ${ }^{4}$

Pour les deux provinces en général, tous les indicateurs restent favorables pour un développement harmonieux du secteur touristique, dont la position géographique, le passé historique, la diversité des composantes humaines, culturelles et naturelles qui sont autant d'atouts les plaçant comme destinations touristiques sahariennes de premier choix. Le produit touristique est réparti en cinq principaux thèmes, à savoir :

- Le tourisme culturel,

- Le tourisme oasien et du désert,

- Le tourisme de montagne,

- Le thermalisme et les cures de bain de sable,

- Ainsi que le tourisme lier à l'industrie cinématographique.

En général les ressources naturelles et humaines qu'offre la zone du sud intérieur constituent une matière première de l'aménagement touristique et un élément de choix des espaces afin de les développer touristiquement, de même qu'une justification de l'implantation des infrastructures touristiques.

${ }^{4}$ Cette stratégie s>articule autour de six chantiers principaux.

- $\quad 1^{\mathrm{er}}$ porte sur la communication et la promotion d'Ouarzazate.

- $\quad 2^{\text {ème }}$ concerne le développement du concept «one stop shop». Ce concept permet à Ouarzazate de devenir l'unique interlocuteur du producteur en lui offrant tous les services de pré production, de production et de post-production nécessaires à la conception de son film.

- $\quad 3^{\text {ème, }}$ vise le recensement des compétences et la formation, est destinée à valoriser le niveau de l'ensemble des techniciens et à aider à l'émergence de nouvelles compétences non existantes pour l'instant (scénaristes, truquistes...),

- $\quad 4^{\text {ème }}$ consiste à instaurer une veille concurrentielle avec la réalisation régulière d'enquêtes sur les pays concurrents.

- $\quad 5^{\text {ème }}$ repose sur la mise en place d'une infrastructure englobant les équipements dédiés aux tournages, à la santé, aux télécommunications et à l'animation sur place.

- $\quad 6^{\text {ème }}$ porte sur la mise en place d'un système d'incitation financière à offrir aux maisons de production, à travers l'octroi d'aides fiscales à l'implantation dans la région et la simplification des procédures douanières pour les importations temporaires de matériel cinématographique. D'où la possibilité de faire passer le cinéma du stade artisanal à une véritable industrie. 
Mais d'autres facteurs interviennent pour fixer les implantations des infrastructures touristiques.

\section{2 - Collectivités locales et leur rôle dans l'animation touristique}

Actuellement, nul ne peut contester le rôle des collectivités locales au niveau de l'aménagement de leurs espaces, et des stratégies de développement qu'elles comptent concrétiser. Néanmoins, ces initiatives et les prises de décisions adéquates diffèrent selon les individus et les collectivités. Pour évaluer le rôle de ces organismes et le degré de différenciation entre les deux espaces, nous nous sommes focalisé sur deux éléments : d'une part la disponibilité $\mathrm{du}$ foncier et $\mathrm{d}$ 'autre part les initiatives individuelles pour faciliter la procédure administrative.

\section{- Le Foncier: un réel atout à Ouarzazate}

L'un des principaux blocages du développement touristique au Maroc au niveau local est représenté par la contrainte du foncier. L'intervention des collectivités locales peut être décisive pour lever entièrement ou partiellement ce blocage. Les communes peuvent ainsi délimiter des sites dits «sites de projets touristiques » afin d'attirer les investisseurs privés.

Dans le Drâa et le Tafilelt, la quasi-totalité des communes locales ont fourni des efforts pour rendre disponibles des terrains réservés aux entrepreneurs en général et à ceux du secteur touristique en particulier.

Cette mesure foncière a été prise à Ouarzazate dès la fin des années soixantedix. Avec le décret de vente du 4 août 1979, la commune urbaine d'Ouarzazate est devenue propriétaire de 237ha 38a, auxquels elle ajoute 14ha selon le décret de vente $1981^{5}$. Ces terrains appartenaient au début aux communes Ahl Ouarzazate et Aït Zineb et ont été transférés à la Municipalité pour des prix symbolique ${ }^{6}$ et ce en deux phases :

- La première acquisition a concerné 251 ha acquis auprès des communes mères d'Ouarzazate.

- La deuxième acquisition a concerné 17.000 ha auprès des communes Ahl Ouarzazate et Ait Zineb.

Par ailleurs des terrains appartenant à l'administration de la défense et situés à l'intérieur du périmètre urbain ont fait l'objet en 1985 d'un échange avec la

\footnotetext{
5 Source : Municipalité d'Ouarzazate - 1995

6 Le prix de vente selon les dossiers de la municipalité d'Ouarzazate est entre de 0,20 Dh et $1 \mathrm{Dh} / \mathrm{m}^{2}$
} 
commune urbaine qui est devenue propriétaire, contre des terrains situés à côté de l'aéroport sont cédés à l'armée.

De ce fait la municipalité devient le principal propriétaire des terrains à Ouarzazate (69\% des terrains) suivie de l'Etat $(20 \%)$, ce qui lui donne une certaine aisance dans la maîtrise du foncier. Elle en réserve une bonne partie aux projets touristiques et à la disposition des investisseurs à des prix symboliques.

Par contre à Errachidia, il faudra attendre le milieu de la décennie quatrevingt, suite au discours royal d'Erfoud et aux conclusions d'un colloque tenu le 20 août 1986 et dont les travaux ont porté sur le tourisme à Erfoud, pour que les élus et autorités envisagent de prendre des mesures dans ce sens. Outre ce retard, les terrains mis à la disposition des investisseurs à Errachidia et à Erfoud manquaient d'équipements et sont trop éloignés de la ville (cas d'Erfoud).

Les initiatives des collectivités locales ont joué donc un rôle décisif dans le choix porté par les investisseurs sur Ourazazate au détriment d'Errachidia, ce qui explique la localisation préférentielle dans le premier pôle. Reste à comprendre le pourquoi de cette différence d'attitude dans le comportement des élus des deux villes. D'où l'importance du rôle des acteurs locaux.

D'autres nouveaux sites sont récemment apparus : quatre zones d'une superficie globale de près de 1000 hectares ont été identifiées par l'agence urbaine d'Ouarzazate et Zagora comme étant ouvertes à l'urbanisation. Ces zones se situent au niveau des municipalités de Zagora et Ouarzazate et à la commune rurale de Tarmigt. Ces terrains collectifs sont répartis en une zone touristique (76 ha), une zone d'industrie du cinéma (120ha), une zone de réserve foncière (337 ha), une zone résidentielle (391 ha) et une zone industrielle (40 ha).

- Initiatives privées : le rôle décisif des acteurs locaux

Le dynamisme touristique que connaît le Sud intérieur en général et Ouarzazate en particulier se renforce aussi grâce aux initiatives privées et au rôle des acteurs locaux. Dans le Sud intérieur de nombreuses collectivités locales ont réservé des terrains afin d'encourager les projets touristiques mais ces terrains tombent dans l'oubli. En réalité l'initiative individuelle est décisive. Il s'agit dans le cas d'Ouarzazate du rôle important joué par le Gouverneur pendant les années quatre-vingt et le début des années quatrevingt-dix en se basant essentiellement sur la simplification des procédures administratives qui entravent toute initiative privée surtout au niveau de l'élaboration du dossier d'investissement «Guichet Unique».

Cette expérience qui a vu le jour à Ouarzazate est à l'origine de la 
concrétisation de la plupart des projets. Par contre la province d'Errachidia est sérieusement pénalisée par les procédures administratives qui entravent encore toute initiative privée. De plus certains éléments s'ajoutent pour aggraver la situation. La mentalité locale montre une grande réticence visà-vis des projets qui contribuent à la pollution de la vie traditionnelle du Tafilelt. En réalité ceci traduit les comportements de certaines catégories sociales qui veulent réserver et «protéger» la vie traditionnelle (Chorfa, et grands commerçants du Tafilelt), ainsi que l'incapacité des responsables et acteurs locaux à jouer leur rôle.

\section{3- Proximité des grands pôles touristiques}

De sa position géographique marginale, le sud intérieur reste à l'écart des flux touristiques nationaux et internationaux. Ce qui aggrave cette situation $c^{\prime}$ est le manque d'une infrastructure routière adéquate qui s'ajoute à une limite au niveau des portes d'entrées. L'analyse du produit touristique du sud intérieur montre que sa fréquentation se fait d'une manière indirecte. La région apparaît comme étant un arrière-pays, un espace de passage des excursions programmées à partir de Marrakech, d'Agadir et de Fès-Meknes. En fait, l'utilisation de la voie terrestre reste depuis longtemps et dans la majorité le seul moyen pour pénétrer à la région.

En général la fréquentation du produit touristique du sud intérieur se réalise dans le cadre des excursions à partir des villes impériales .

Le poids de la province d'Ouarzazate dans ces excursions reste très important si on la compare à d'Errachidia. Cependant Ouarzazate reçoit déjà 13,8\% des flux touristiques visitant la région et arrivant directement par voie aérienne. Pour la première fois de son histoire Ouarzazate a dépassé le seuil des 100.000 passagers sur l'année 2007. Ce sont des touristes qui programment la région surtout pendant les vacances d'une semaine et préfèrent séjourner dans le sud intérieur uniquement. Ceci nous amène à conclure que la ville d'Ouarzazate tend à devenir la seule station de séjour du Sud intérieur et qu'Errachidia constituera probablement son arrière-pays.

L'émergence de la dimension culturelle dans le tourisme marocain nécessite la définition d'un produit touristique «Sud intérieur» autonome et avec accès direct. Seule Ouarzazate a connu un début de désenclavement avec l'inauguration de son aéroport en 1986 et l'ouverture des vols directs ParisOuarzazate, ce qui a encouragé les investisseurs à s'y implanter. Mais malgré cela sa fréquentation plus élevée que celle d'Errachidia est avant tout liée à sa proximité des deux plus grandes stations touristiques du Maroc : Agadir sur la côte et Marrakech à l'intérieur. Elle reçoit de ce fait le trop- 
plein de ces deux villes dans le cadre d'excursions et n'arrive pas à s'imposer totalement comme une destination autonome. Errachidia est lié au binôme Fès-Meknes qui est moins dynamique que le binôme Marrakech-Agadir et ceci se répercute sur la fréquentation de la partie orientale du Sud intérieur. En outre, l'aéroport d'Errachidia malgré son ouverture au trafic civil en 1996 est peu fréquenté par les mouvements réguliers des vols et répond surtout à des besoins militaires et particuliers.

\section{Les impacts du tourisme : enjeux environnementaux}

\section{Impacts socio-économiques du tourisme}

Les impacts touristiques peuvent être identifiés selon trois axes :

La dimension sociale: emploi touristique: trois niveaux ont été distingués: emploi dans le secteur d'hébergement qui emploie la quasi-totalité de la main d'œuvre, l'emploi dans le secteur para-hôtellerie et enfin l'emploi non organisé. Parmi les caractéristique de ce personnel surtout dans le secteur hôtelière: une main d'œuvre jeune mais non qualifiée et non spécialisée.

La dimension économique: l'intégration du tourisme dans l'environnement local

L'activité touristique pose des difficultés concernant la manière avec laquelle on peut mesurer son intégration dans la vie locale. En général, l'importance du secteur touristique dans le développement économique de la région reste encore faible. Dans ce sens, la faiblesse des ressources locale joue parfois négativement dans le degré de l'intégration du secteur touristique. Une situation qui pousse les investisseurs de s'approvisionner en dehors de la région. Ce qui ne permit pas de bénéficier énormément des activités touristiques.

$C^{\prime}$ est dans ce sens, que cet article vise essentiellement la valorisation du patrimoine à des fins touristique afin de mieux profiter aussi bien des atouts et la richesse de cette région et des activités touristiques : De ce fait, il convient de vendre la région comme un lieu de séjour et non plus seulement comme un lieu de passage.

La dimension spatiale : la consommation de l'espace par les activités touristique La consommation de l'espace et son organisation par les activités touristique dans le sud intérieur a fait ressortir quatre comportements :

- Activités touristiques qui se localisent à la marge des centres urbains : cas des 
petits centres de Tinghir - Boumalen et Kelaa M'Gouna

- Vers la concentration et l'organisation de l'espace : cas de la ville de Ouarzazate - Des établissements touristiques qui cherchent l'isolement mais au détriment des espaces agricoles : cas de Zagora. Ce comportement peut généraliser pour tous les autres unités dans le milieu rural : les auberges au long de Merzougua massif de m'goun - Ait Ben Haddou...

- Activités touristiques qui renforcent et véhiculent l'accroissement urbain : cas du centre d'Erfoud

\section{Enjeux environnementaux : consommation de l'eau}

Avec les nouvelles implantations touristiques aussi bien en zone urbaine et dans les différents coins du milieu rural, le problème de l'enjeu environnemental se pose. L'un des aspects les plus préoccupants dans l'évolution du tourisme au Sud intérieur reste la question de l'environnement naturel et de sa dégradation: dégradation des sites (gorges - dunes de sables, pollution des rivières, dégradation des ressources en eaux...) tels sont les problèmes les plus préoccupants.

En effet, le développement du tourisme de randonnée et du tourisme caravanier, du tourisme rural en général s'accompagne de la diffusion des déchets au fond des montagnes et jusqu' aux dunes les plus reculées: Merzouga - tinfou - chegaga...

A travers les résultats de nos travaux de doctorat national (2007) consacrée à la consommation de l'eau par les activités touristiques dans les oasis du sud et plus précisément dans le Haut Draa, il ressort ce qui suit :

- Une croissante des activités touristiques (demande en eau) contre une diminution et la rareté des ressources en eau;

- Une volonté de développer un tourisme intégré dans un espace oasien fragile,

- Diminution progressive des réserves en eau selon les années;

En effet, les apports moyens annuels au barrage Mansour Ed-Dahbi s'élève à $415 \mathrm{Mm} 3$, sans pour autant ignorer la non régularité de ces apports :

- 375 Mm3 entre 1973-1994/an

- $\quad 75 \mathrm{Mm} 3$ entre 1982-1994/an

- $1125 \mathrm{Mm} 3$ 1987-1990/an 


\begin{tabular}{|c|c|c|c|}
\hline Oued & Station & Bassin versant & Apport moyen Mm3/an \\
\hline Dades & Ait Mouted & 1525 & 105 \\
\hline M'Goun & Ifree & 1239 & 128 \\
\hline Dades & Tinouar & 6680 & 242 \\
\hline Ouarzazate & Tifoultoute & 3507 & 134 \\
\hline Douchène & Assaka & 1387 & 13 \\
\hline \multicolumn{3}{|c|}{ TOTAL } & $\mathbf{6 2 2}$ \\
\hline
\end{tabular}

Tableau 1 - Apports des oueds du bassin versant du Haut Draa

Concernant la demande en eau potable en générale et en particulier celle du secteur touristique, il est important de souligner qu'elle ne dépasse pas les $30 \%$ de la consommation totale surtout que la consommation domestique reste en tête; mais cette demande se concentre essentiellement en saison du printemps (32\% de la consommation en eau potable / $40 \%$ des nuitées touristiques) suivi par une deuxième pointe en été comme illustre les graphiques ci-après :

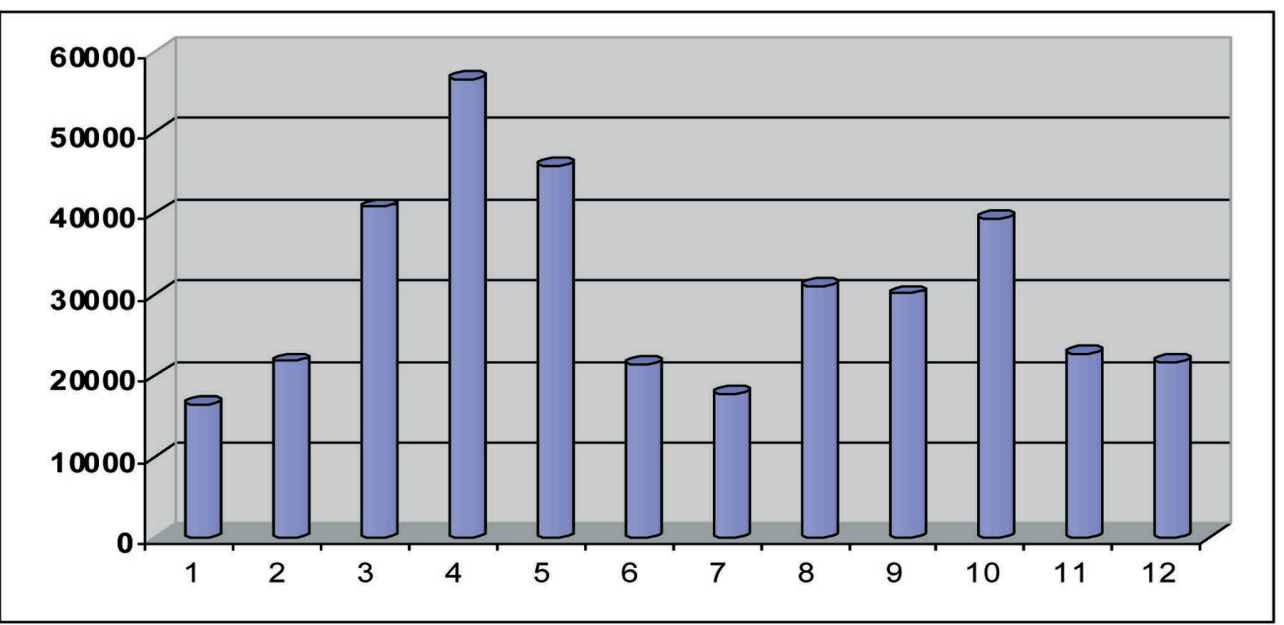

Fig. 4 - Consommation mensuelle de l'eau potable par les unités hôtelières (m3) (2000)

Selon les catégories hôtelières, les besoins en eau pour une unité se présentent comme suit :

- Hôtel 5*

$: 68503 \mathrm{~m} 3 / \mathrm{an}$

- Résidence touristique : $47040 \mathrm{~m} 3 / \mathrm{an}$

- Hôtel 1* : $6452 \mathrm{m3} / \mathrm{an}$

- Café-restaurant : $670 \mathrm{~m} 3 / \mathrm{an}$ 


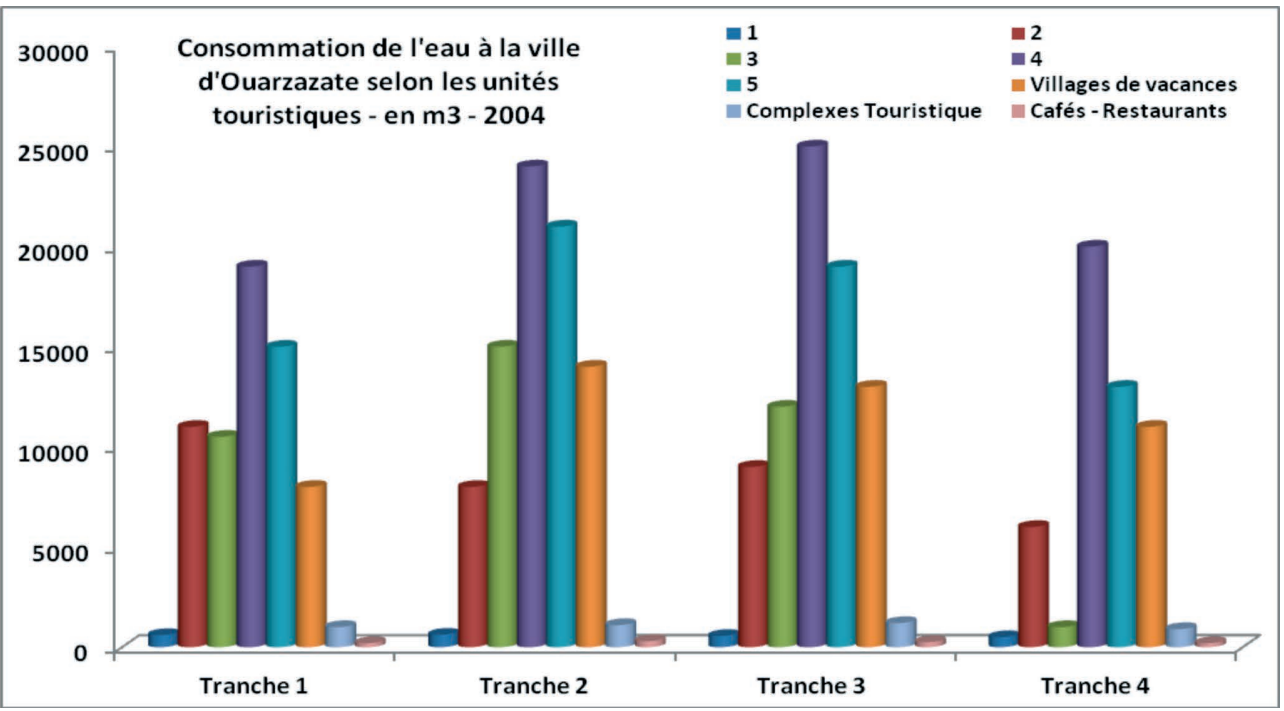

Fig. 5 - consommation de l'eau à la ville d'Ouarzazate selon les unités touristiques (m3) en 2004

Concernant les équipements dont disposent les unités d'hébergement surtout les hôtels :

- $\quad 36 \%$ des unités touristiques hôtelière possèdent une piscine ;

- $\quad 70 \%$ des unités d'hébergement touristiques possèdent des réservoirs d'eau,

- $\quad 85 \%$ d'hébergement touristiques ont des puits.

V

La concentration mensuelle et l'évolution des quantités consommées par le secteur touristique reste une caractéristique de ce secteur surtout dans un espace oasien aride comme indique les projections de la consommation par secteur jusqu'en 2010.

Il parait donc que la croissance de la consommation touristique au fil des années surtout dans le cas du pôle touristique dans la région (ville d'Ouarzazate) connaît une croissance qui mérite d'être prise en considération par les décideurs:

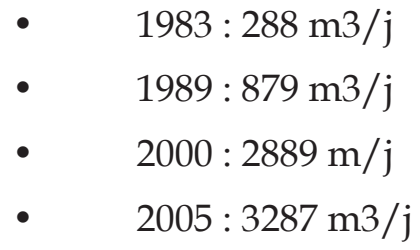




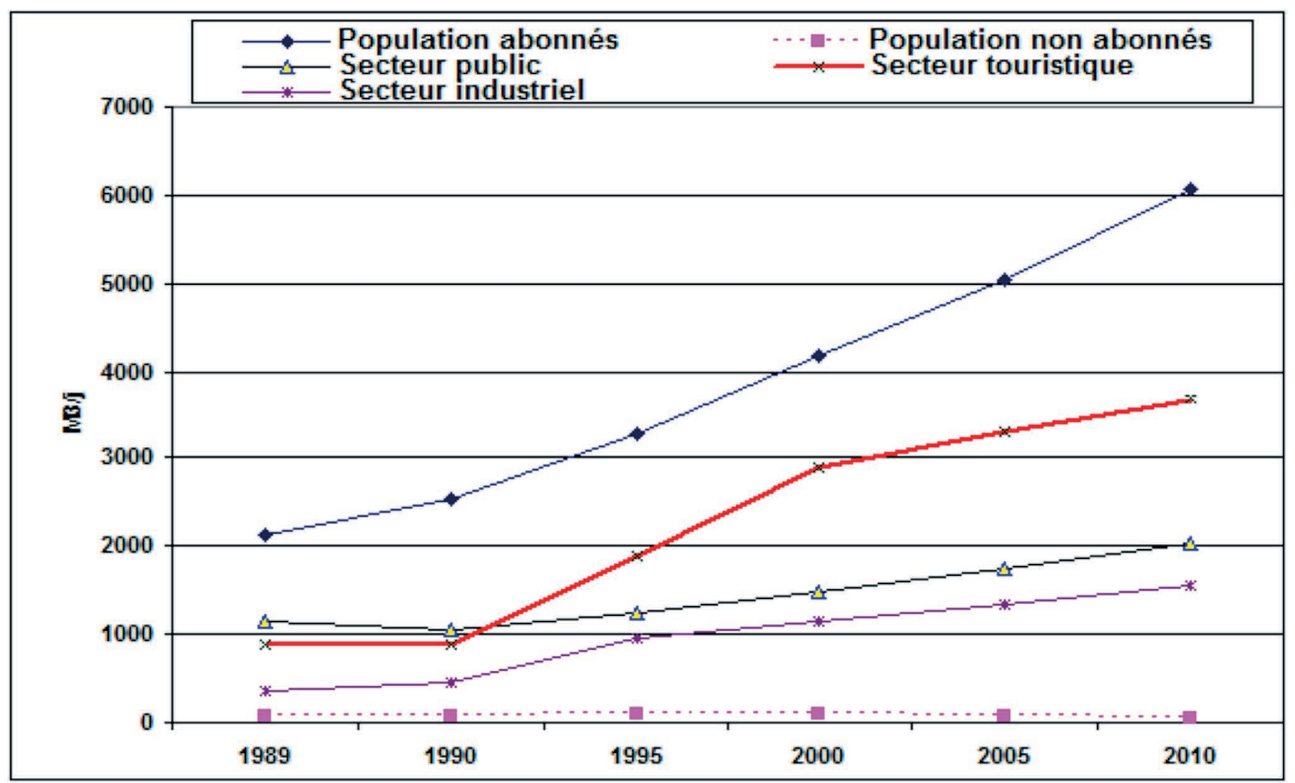

Fig. 6 - Projection de la consommation de l'eau potable selon les secteurs

\begin{tabular}{|l|c|c|}
\hline \multicolumn{1}{|c|}{ Centre } & $\mathbf{2 0 0 5}$ & $\mathbf{2 0 2 0}$ \\
\hline Ouarzazate Tabounte & 3.2 & 8.4 \\
\hline Skoura & 0.1 & 0.2 \\
\hline Kelaa M'Gouna & 0.2 & 0.9 \\
\hline Boumalene & 0.4 & 1.2 \\
\hline Taznakhte & 0.1 & 0.3 \\
\hline Total & 4 & 12 \\
\hline Besoin milieu rural & 2.2 & 5 \\
\hline Total général & 6.2 & $17 \mathrm{Mm} 3$ \\
\hline
\end{tabular}

Tableau 2 - La demande en eau potable par centre en Haut Draa (en Mm3/an)
Ce qui représente environ 1,1 Mm3/ an que le secteur touristique consomme seul; en raison aussi des futurs projets touristiques et de leurs grandeurs qui dépassent parfois les 400 et 500 lits. La consommation hôtelière représente $70 \%$ de la consommation touristique en général.En fait, il est important de souligner que le grand consommateur de l'eau actuellement est le secteur agricole car le volume d'eau destiné à l'usage agricole s'élève à $280 \mathrm{Mm} 3 / \mathrm{an}$, soit $139 \mathrm{Mm} 3$

venant des eaux superficielles et $41 \mathrm{Mm} 3$ des eaux souterraines.

Par ailleurs, le taux de desserte en eau potable de la population rurale a augmenté de $14 \%$ en 1994 pour atteindre $91 \%$ en 2007 . Cette situation illustre l'augmentation et la pression envers les ressources en eau ainsi que les problèmes que connaît l'alimentation en eau potable dans les oasis du sud (Fig.7). 
Et malgré cette série de problèmes, les enquêtes sur terrain ont permis de relever deux opinions différentes :

\begin{tabular}{|l|}
\hline Problème naturel : faiblesse des préci pitations et aridité du climat ; \\
$\begin{array}{l}\text { Problèmes socio-économiques : manque d'infrastructures et } \\
\text { augmentation des couts de creusement de puits; }\end{array}$ \\
$\begin{array}{l}\text { Diminution progressive de la nappe phréatique (17\%) et sèchement } \\
\text { des khettaras; }\end{array}$ \\
$\begin{array}{l}\text { Perte de la main d'œuvre agricole et omission des moyens } \\
\text { traditionnels: mobilisation des eaux; }\end{array}$ \\
Dégradation des oasis; \\
Qualité de l'eau : $21 \%$ de la population locale déclare ce problème ; \\
Coupures progressives de l'eau potable surtout en été. \\
\hline
\end{tabular}

Fig. 7 - Dynamique des ressources en eau

- Une vision optimiste des autorités locales surtout les services concernés par la mobilisation des eaux qui affirme que la région dispose des quantités d'eau suffisantes et que la crise de l'eau ne se pose pas.

- Une vision pessimiste de la population locale qui ne cesse de déclarer la crise de l'eau dans la région.

C'est vrai, selon les prévisions du bilan des ressources et les besoins en eaux dans le bassin Sud - Atlasique en général, la région va connaître un déficit d'ici 2020 comme l'illustre le tableau 3.

\begin{tabular}{|c|c|}
\hline Années & Déficit \\
\hline 2000 & -49 \\
\hline 2010 & -151 \\
\hline 2020 & -94 \\
\hline
\end{tabular}

Tab. 3 - Disette en eau dans la zone
Selon les prévisions du programme d'alimentation en eau potable du monde rural (PAGER), les besoins du milieu rural dans la province d'Ouarzazate s'élève à $15291 \mathrm{~m} 3$ /

jours, soit 5,6 Mm3/an à l'horizon 2022. Actuellement, ils sont de l'ordre de 2,5 Mm3/an et celles du secteur agricole $213 \mathrm{Mm} 3 /$ an contre $180 \mathrm{Mm} 3 /$ an actuelle.

Cependant, concernant, le secteur touristique, et à travers les résultats de nos recherches, nous pouvons affirmer qu'à ce stade, le secteur touristique ne présente pas un facteur de dégradation des ressources en eau. Mais ce qui est remarquable c'est que le secteur touristique représente un élément qui s'ajoute aux autres secteurs traditionnels concernant la consommation de l'eau.

Cela ne signifie pas que le secteur touristique ne présente pas un facteur de dégradation de l'environnement, mais il est temps d'élaborer une stratégie pour développer un tourisme intégré dans l'environnement oasien très 
fragile et a des caractéristiques spécifiques en respectant les valeurs de la rareté des ressources en eau.

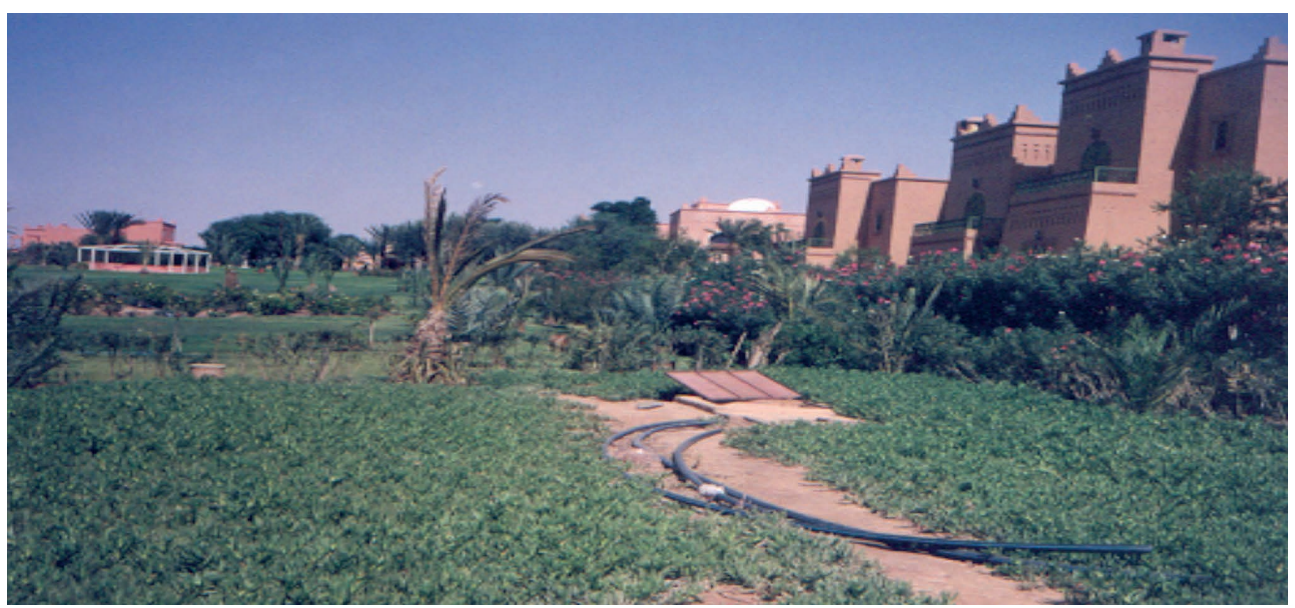

Photo 1 - Lotissement des amis - Lac du barrage Manssour Ed-Dahbi - Ouarazate

Dans un souci d'efficacité économique, le tourisme constitue un secteur très important à développer, mais n'est pas au détriment des valeurs locales, qui constituent en premier lieu les atouts du produit touristique.

VII. PAT « désert \& Oasis ». Ouarzazate, Zagora et Errachidia: 1'une des solutions pour un développement touristique intégré «version 2010»

Le projet de Pays d'accueil touristique (PAT) a été lancé en 2007, avec l'objectif visant de contribuer à la promotion et la valorisation du tourisme rural, un créneau des plus prometteurs destiné à l'organisation de l'activité touristique en milieu rural.

Cet ambitieux projet s'assigne pour objectifs, de répondre au mieux à la demande croissante de la clientèle nationale et étrangère et surtout d'organiser l'offre et d'améliorer la qualité du service.

Ce projet constitue l'un des axes stratégiques de la nouvelle politique touristique dans le cadre de la vision 2010, et se décline en mesures opérationnelles et ambitieuses touchant à tous les créneaux de développement de l'activité touristique en milieu rural (accueil, information, hébergement, environnement, promotion, commercialisation du produit, mise en valeur des produits du terroir).

Cette initiative vise à créer de la richesse pour les populations locales, de 
conforter et de compléter le projet de développement du tourisme et de rééquilibrer le développement touristique régional. La proximité d'une zone d'émission de touristes, l'adhésion des acteurs locaux et l'existence d'une activité du tourisme rural sont autant d'atouts sur lesquels s'appuie ce projet ambitieux.

\section{Conclusion}

En guise de conclusion, le Drâa et le Tafilalet présente des destinations touristiques qui, ont été lancées sur le marché du tourisme international en même temps, et elles ont connu deux évolutions différentes. Alors qu'Ouarzazate tend à devenir un véritable pôle de tourisme avec un produit spécifique, Errachidia a du mal à démarrer. Des raisons multiples expliquent ce décalage, mais le rôle des acteurs locaux semble être décisif.

On peut s'interroger sur le devenir de ces deux pôles qui se retrouvent désormais groupés et localisés dans la même région économique selon le nouveau découpage de 2015. Ceci fut déjà le cas dans les découpages précédents, mais aujourd'hui, plus que jamais la région est vue comme un sérieux outil de développement en raison des attributions et des objectifs fixés.

Par ailleurs, concernant les impacts environnementaux, la consommation de l'eau par le secteur touristique, constitue un élément qui s'ajoute aux secteurs traditionnels, mais ne constitue pas un élément de dégradation de l'environnement mais il est temps d'élaborer une stratégie efficace pour un développement durable et respecter la notion du tourisme intégré dans les régions oasiennes.

Enfin, et dans le cadre de développer un tourisme intégré et durable, il est temps de sensibiliser les acteurs, les partenaires, les autorités locales, et la population afin d'établir un plan d'action permettant le développement de la région en général et d'un tourisme orienté "Nature, rural et ses différentes facettes » afin de distinguer l'originalité de cette destination afin de rentabiliser ce secteur. A ce sujet des axes méritent d'être souligner et en tant que conditions de succès :

- Doter la région en infrastructures routière afin de désenclaver les localités et permettre un développement harmonieux;

- Implications de tous les acteurs du tourisme pour la durabilité du secteur : rendre le tourisme durable là où il se vit, $c^{\prime}$ est à dire dans les territoires, dans les localités, et auprès de ceux qui le font vivre, les opérateurs et les touristes, 
les populations elles-mêmes tout comme les gestionnaires de ces territoires : tous le monde doit remplir ses obligations de base en matière de service public.

- Tous les acteurs doivent travailler ensemble et un grand effort doit être donné à la gouvernance locale. Les acteurs locaux doivent devenir les catalyseurs du développement de leurs territoires ;

- Valoriser la richesse culturelle de la région en s'inspirant des expériences réussies dans d'autres pays ;

- Planification des projets : La planification consiste à mettre au point un mécanisme de gestion durable propre à chaque site, en termes d'objectifs, d'actions de conservation, de budgétisation, de recherches et de documentation :

- Identification et inventer la richesse patrimoniale : humaine et naturel;

- Evaluer selon les priorités et en fonction des budgets disponible;

- Volet juridique et institutionnel - convention

- Mise en œuvre :

- Réhabilitation - gestion et la conservation des sites du Patrimoine et leurs utilisation ;

- Suivi et évaluation ;

- Recherche de financement : Crier un fond permettant la mise en place des projets éco-tourisme, notamment dans les zones reculées dont le capital naturel peut constituer une source d'emplois et en vue d'identifier les possibilités de financement durables;

- Créer des produits touristiques spécifique à la zone;

- Adopter un plan en matière de sauvegarde du patrimoine culturel et son utilisation à des fins économique;

- Elaborer des outils statistiques permettant d'évaluer et de suivre l'impact du tourisme sur les ressources en eau et sur l'environnement.

\section{Bibliographie}

Oujamaa Abderrahmane, 1999, Tourisme et l'espace - cas de Ouarzazate et Errachidia - entre le choix des décideurs et la réalité actuelle, Thèse de Troisième Cycle (DES), Université Mohamed V - Faculté des Lettres et des Sciences Humaines - Rabat, Unité de formation et de recherche "Aménagement de l'espace régional»,pp 410.

Oujamaa Abderrahmane, 2007, Activités touristiques et consommation de l'eau dans les oasis du sud : cas Haut vallée du Draa, Doctorat National, Université Hassan II - 
Faculté des Lettres et des Sciences Humaines - Mohammadia, Unité de formation et de recherche «Eau et Civilisation », pp 310.

Oujamaa Abderrahmane, 2013, le tourisme intérieur français de la période du Protectorat au Maroc, précurseur et initiateur du tourisme culturel, cas du Sud du Maroc : La Province d'Ouarzazate, in L'héritage colonial du Maroc ; Herbert Popp \& Mohamed Aït Hamza (éd.) (page 289-306). 2013.

Pearce. D, 1987, Géographie du tourisme, collection Géographie, Nathan, p 172.

Bernadette Mérenne-Schumaker, 1996, La localisation des services, collection Géographie, NATHAN.

El Meskin. M., 1993, Les Filala entre le Ksar et la ville, contribution à l'étude des migrations internes à partir du Tafilalt (Sud-Est du Maroc), thèse de doctorat de 3ème cycle, Université de Toulouse- le Mirail, U. F. R. Géographie-Aménagement, Institut Daniel Faucher

F Boubakraoui. My. Hassan, 1994, Le Tafilalet et l'évolution économique et social d'un espace Sud-Marocaine, Thèse de Doctorat, nouveau régime, Université de Genève.

G Terrasse. H., Kasbas, Berbère de l'Atlas et des oasis, les grandes architectures du Sud Marocain, Editions des Horizon de France, Paris, p. 70-80.

Royaume du Maroc, Ministère de l'Urbanisme, de l'Habitat, du Tourisme et de l'Environnement, 1978, Aménagement touristique-région Ouarzazate-Errachidia.

Royaume du Maroc, Ministère de l'intérieur, Province d'Ouarzazate, Etude d'aménagement du site du Lac Mansour Ed-Dahbi, 1991 - 1993. 